\title{
Comparative Analysis of the Genomes of Three Field Isolates of the Rice Blast Fungus Magnaporthe oryzae from Southern China
}

\author{
Kailing Chen*, Jinqi Feng*, Shen Chen, Jing Su, Jianyuan Yang, Congying Wang, Aiqing Feng, \\ Bing Chen, Xiaoyuan Zhu\#, Wenjuan Wang\# \\ Guangdong Provincial Key Laboratory of High Technology for Plant Protection, Plant Protection Research Institute, \\ Guangdong Academy of Agricultural Sciences, Guangzhou, China \\ Email: "wangwenjuan@gdppri.com, "ricedisease@gdppri.com
}

How to cite this paper: Chen, K.L., Feng, J.Q., Chen, S., Su, J., Yang, J.Y., Wang, C.Y., Feng, A.Q., Chen, B., Zhu, X.Y. and Wang, W.J. (2021) Comparative Analysis of the Genomes of Three Field Isolates of the Rice Blast Fungus Magnaporthe oryzae from Southern China. Agricultural Sciences, 12, 713 725.

https://doi.org/10.4236/as.2021.127046

Received: February 19, 2021

Accepted: July 4, 2021

Published: July 7, 2021

Copyright $\odot 2021$ by author(s) and Scientific Research Publishing Inc. This work is licensed under the Creative Commons Attribution International License (CC BY 4.0).

http://creativecommons.org/licenses/by/4.0/

(c) (i) Open Access

\begin{abstract}
Rice blast caused by Magnaporthe oryzae (M. oryzae) is one of the most destructive diseases, which causes significant rice yield losses and affects global food security. To better understand genetic variations among different isolates of $M$. oryzae in the nature field, we re-sequenced and analyzed the genomes of three field isolates, QJ08-2006, QJ10-10, and QJ10-3001, which showed distinct pathogenicity on Xin-Yin-Zhan, an elite variety in South China. Genome annotation indicated that these three isolates assemblies have similar genome sizes with $38.4 \mathrm{Mb}, 38.3 \mathrm{Mb}$, and $38.4 \mathrm{Mb}$, respectively. The QJ08-2006 assembly has 2082 contigs with an N50 of $127.4 \mathrm{~kb}$, the QJ10-10 assembly has 2239 contigs with an N50 of $105.13 \mathrm{~kb}$, the QJ10-3001 assembly has 2025 contigs with an N50 of $133.16 \mathrm{~kb}$. A total of 10,432 genes including 1408 putative secreted protein genes were identified from the annotated isolate QJ08-2006 genome, 10,418 genes including 1410 putative secreted protein genes were identified in QJ10-10, and 10,401 genes including 1420 putative secreted protein genes were identified in QJ10-3001. There are as many as 11,076 identical genes in these three isolates and contained only a few unique genes among three isolates, of which 277 unique genes in QJ08-2006 and 264 unique genes in QJ10-10, and 213 unique genes in QJ10-3001. Most of the predicted secreted protein genes had been identified, and the three re-sequenced strains contained 371, 369, and 387 small Indel, respectively. Avr genes were analyzed in several sequenced Magnaporthe strains, the results revealed that $A v r-P i 9$ and $A v r$-Piz- $t$ were present in all the sequenced isolates. The isolates QJ08-2006 contained AvrPib, QJ10-10, and QJ10-3001 had an insertion of a Pot3 element in the promoter of the $A v r P i b$ gene. Our
\end{abstract}

${ }^{\star}$ K.L., Chen and J.Q., Feng contributed equally to this work.

\#Corresponding author. 
results showed that, the rapid dominancy of virulence mutant isolates via clonal propagation displayed in the field after the release of the elite variety Xin-Yin-Zhan.

\section{Keywords}

Rice Blast, Magnaporthe oryzae, Comparative Genomics, Genetic Variation, Secreted Protein Genes

\section{Introduction}

Rice (Oryza sativa) is one of the major staple food crops for more than half of the human population worldwide [1]. However, a large portion of yield is lost due to agricultural disease and pests annually [2]. Rice blast, caused by the filamentous ascomycete fungus Magnaporthe oryzae (syn. Pyricularia oryzae), occurs in every rice-growing regions in worldwide [3] [4], and poses a major threat to global food security [5] [6]. This pathogen can infect leaves, stems, panicles, nodes, and even roots of rice at the whole stage of development and also infects wheat and other small grains [7] [8] [9]. As genomes of $M$. oryzae have been sequenced and the data of the genomes is available to the public [10], rice and $M$. oryzae have been developed as the classical model system for studying the plant-microbe interactions [11].

In many parts of the world, deployment of disease-resistance varieties is an economical and efficient strategy for the management of rice blast [2]. However, M. oryzae often can overcome rice resistance based on race-specific resistant genes [12]. New resistant varieties often lose their resistance within a few years after release to the fields. High genetic variations occur within the field $M$. oryzae populations, especially in the pathogenic traits i.e., secreted pathogen avirulence or effectors evolve rapidly to counteract plant defense, maybe a major challenge to control rice blast disease [13].

The advent of whole-genome sequencing technology and the subsequent deployment of the next generation sequencing (NGS) technology, genome re-sequencing, comparative studies, and the deployment of PacBio sequencing technology, has been reported in M. oryzae in the last decade [14] [15]. More than 200 isolates of $M$. oryzae have been successfully generated genome assemblies using high throughput genome sequencing technologies [10] [14] [15]. The genome of the laboratory $M$. oryzae strain 70 - 15 was the first to be sequenced among plant-pathogenic fungi using the Sanger sequencing method [10]. Subsequently, multiple $M$. oryzae field isolates have been sequenced using NGS technology. Such as, field isolates from Japan (P131 and Ina168) and China (Y34) were sequenced using 454 sequencing technology [16] [17]. More recently field isolates FJ81278, HN19311, and 98-06 from China, isolates B157, MG01, and RML-29 from India, and isolates R88-002, Ro1-1, and V86010 from the Philippines, and 
100 isolates of $M$. oryzae ( $P$. oryzae) from China as well as several different regions around the world have been sequenced using Illumina high-throughput sequencing technology [15] [18] [19] [20] [21] [22]. To generate near-complete genome assembly for $M$. oryzae field isolates and evaluated the possible contribution of TEs to genomic variation events and virulence-associated secreted proteins polymorphism, field isolates FJ81278 and Guy11 have been sequenced by PacBio sequencing technology [14]. The comparison genomics analysis reported some isolate-specific genomic regions, genes under diversifying selection, hundreds of unique genes, and the specific events in the evolution of field populations of $M$. oryzae [15] [17]. These reports suggest the NGS technology is a powerful tool to elucidate the molecular basis of virulence, study pathogen genome variation, and provide a new method for cloning novel avirulence genes.

Genome-wide studies of more blast field isolates and identification of novel $A v r$ effectors will help researchers to further disclose the mechanism of pathogen and plant co-evolution. QJ08-2006, QJ10-10, and QJ10-3001 were three field $M$. oryzae isolates in South China, which showed different pathogenicity on a released resistant variety Xin-Yin-Zhan. In this study, we investigated the genomic variations and DNA polymorphisms of three $M$. oryzae field isolate by NGS technology in an attempt to understand the genomic variation within field isolates after release of the resistant variety. This work will be useful for breeders and pathologists to understand the variation of $M$. oryzae virulences and develop strategies to improve the resistance of varieties against rice blast.

\section{Materials and Methods}

\subsection{M. oryzae Field Isolates Used}

The field M. oryzae isolates QJ08-2006 was collected from cv. Ma-Ba-Yin-Zhan (MBYZ), the isolates QJ10-10, and QJ10-3001 were collected from cv. Xin-YinZhan (XYZ). The newly improved blast-resistant rice variety of XYZ was developed using the susceptible elite variety, MBYZ, as the recurrent line. All these three isolates were collected from the panicle neck of rice plant samples at Guangdong Province, China in the year 2008 and 2010, respectively, and have been kept at Guangdong Academy of Agricultural Sciences (GDAAS, China).

\subsection{Genomic DNA Extraction and Genome Sequencing}

Isolates of $M$. oryzae were cultured in liquid complete medium (CM: 6 g yeast extract, $6 \mathrm{~g}$ casein hydrolysate, and $10 \mathrm{~g}$ sucrose in $1 \mathrm{~L}$ of medium) to prepare the vegetative mycelia for the extraction of genomic DNA [23]. Vegetative mycelia were harvested and ground into a fine powder in liquid nitrogen. The genomic DNA of each $M$. oryzae isolate was extracted following a protocol described [24]. The whole-genome sequencing libraries of the three selected iso- 
lates, QJ08-2006, QJ10-10, and QJ10-3001, were prepared using the Illumina paired-end DNA sample Prep Kit and sequenced by Illumina Hiseq2000 sequencing technology at BGI Tech (Shenzhen, China). Approximately 2 Gb clean reads from the library averaging $500 \mathrm{bp}$ in size were obtained for these three sequenced isolates.

\subsection{Genome Assembling}

Short reads generated were mapped to the $M$. oryzae reference genome 70 - 15 (version 8, Magnaporthe Comparative Sequencing Project, Broad Institute of Harvard and MIT). De-novo genome sequence assembly was conducted with CLC Genomic Workbench 7.0 software.

\subsection{Gene Prediction and Annotation}

The genomic sequences of three re-sequenced isolates were filtered by using Repeatmask 3.3 (http://www.repeatmasker.org, species = "magnaporthe_grisea"), and repeat sequences used were from RepBase (http://www.girinst.org/). Gene predictions were conducted through the Fgenesh model of Molquest (species, magnaporthe, sequence length, more than 20 aa) [21]. Masked genome sequences of QJ08-2006, QJ10-10, and QJ10-3001 were compared using the MUMMER package to construct the chromosome sequence for each isolates, respectively based on the reference 70 - 15 genomic sequence [25]. Protein domain was predicted using SMART database. We used SignalP4.0, Protcomp-AN, and TMHMM2.0 package to predict secreted protein genes of M. oryzae [26] [27].

\section{Results}

\subsection{Genome Sequencing and Assembly of the Genomes of QJ08-2006, QJ10-10, and QJ10-3001}

Genomic DNA of isolates QJ08-2006, QJ10-10, and QJ10-3001 were prepared as paired-end libraries and sequenced by using the Illumina HiSeq2000 system at BGI Tech (Shenzhen, China). In total, $1860 \mathrm{Mb}, 1810 \mathrm{Mb}$, and $1810 \mathrm{Mb}$ of clean data for QJ08-2006, QJ10-10, and QJ10-3001 were generated, respectively, which derived from paired-end sequence libraries with an average fragment length of $500 \mathrm{bp}$ in size (Table 1). The genomes of these three isolates were de-novo assembled using CLC Bio genomic workbench software and annotated using a standard procedure as described previously [28]. As showed in Table 1, these three assemblies resulted in similar genome size of $38.4 \mathrm{Mb}, 38.3 \mathrm{Mb}$, and $38.4 \mathrm{Mb}$, respectively. The QJ08-2006 assembly had 2082 contigs with an N50 of $127.4 \mathrm{~kb}$, the QJ10-10 assembly had 2239 contigs with an N50 of $105.13 \mathrm{~kb}$, the QJ10-3001 assembly had 2025 contigs with an N50 of $133.16 \mathrm{~kb}$. However, the genomic features of these three isolates described herein showed much more distinct in most features from other isolates reported previously, including 98-06, P131, Y34, FJ81278, HN19311, R88-002, and 70-15 (Table 1). 
Table 1. Genome assembly and annotation statistics from ten $M$. oryzae isolates.

\begin{tabular}{|c|c|c|c|c|c|c|c|c|c|c|c|}
\hline & \multirow[b]{2}{*}{ Sequencing platform } & QJ08-2006 & QJ10-10 & QJ10-3001 & $98-06$ & P131 & Y34 & FJ81278 & HN19311 & R88-002 & $70-15$ \\
\hline & & Illumina & Illumina & Illumina & Illumina & $\begin{array}{c}\text { Sanger }+ \\
454\end{array}$ & $\begin{array}{l}\text { Sanger + } \\
\quad 454\end{array}$ & PacBio & Illumina & Illumina & Sanger \\
\hline \multirow[t]{7}{*}{ Genome } & $\begin{array}{l}\text { Assembly genome size } \\
(\mathrm{Mb})\end{array}$ & 38.41 & 38.28 & 38.40 & 45.3 & 37.95 & 38.87 & 43.85 & 37.1 & 39.08 & 40.95 \\
\hline & Coverage (fold) & $50 \mathrm{X}$ & $50 \mathrm{X}$ & $50 \mathrm{X}$ & $135 \mathrm{X}$ & $20 \mathrm{X}$ & $21 \mathrm{X}$ & $247 \mathrm{X}$ & - & 109X & - \\
\hline & Contig Number & 2082 & 2239 & 2025 & 1161 & 2601 & 2114 & 54 & 6249 & - & 219 \\
\hline & $\begin{array}{l}\text { Average contig length } \\
(\mathrm{kb})\end{array}$ & 18.45 & 17.09 & 18.96 & - & - & - & 811.97 & - & - & 186.99 \\
\hline & N50 contig length $(\mathrm{kb})$ & 127.4 & 105.13 & 133.16 & 88.6 & 37.7 & 53 & & 147.4 & - & \\
\hline & $\mathrm{G}+\mathrm{C}$ content $(\%)$ & 51.40 & 51.49 & 51.39 & 50.8 & 51.5 & 51.3 & 51.03 & 51.5 & - & 51.61 \\
\hline & Repeat sequence & $2.28 \%$ & $2.22 \%$ & $2.28 \%$ & $9.30 \%$ & $2.26 \%$ & $2.54 \%$ & $14.43 \%$ & $2.83 \%$ & $2.46 \%$ & $11.10 \%$ \\
\hline \multirow[t]{3}{*}{ Genes } & $\begin{array}{l}\text { Number of predicted } \\
\text { genes }\end{array}$ & 10,432 & 10,418 & 10,401 & 14,030 & 12,722 & 12,869 & 11,927 & 10,256 & 12,344 & 12,440 \\
\hline & Average gene length (bp) & 1532 & 1538 & 1535 & 1410 & 1334 & 1332 & - & 1745 & - & 1356 \\
\hline & $\begin{array}{l}\text { Number of secreted } \\
\text { protein genes }\end{array}$ & 1408 & 1410 & 1420 & 1732 & 1005 & 998 & - & - & 1008 & 1005 \\
\hline
\end{tabular}

\subsection{Genome Annotation and Gene Prediction}

A total of 10,432 genes ( $\geq 20$ aa) including 1408 putative secreted protein genes were identified from the annotated isolate QJ08-2006 genome, a total of 10,418 genes ( $\geq 20 \mathrm{aa}$ ) including 1410 putative secreted protein genes were identified from the annotated isolate QJ10-10 genome, and a total of 10,401 genes ( $\geq 20 \mathrm{aa})$ including 1420 putative secreted protein genes were identified from the annotated isolate QJ10-3001 genome (Table 1). The average length of predicted genes was $\sim 1530 \mathrm{bp}$. Intriguingly, these 3 isolates showed quite similar features, e.g., genome size, number of predicted genes, and secreted protein genes. By contrast, they were distinct from other sequenced isolates in most features (Table 1).

\subsection{Identification of Presence/Absence Variation in Different Isolates}

To identify the unique genes in the three field isolates, the genomic content was compared to each other. To avoid false-positive gene predictions, the genes from each of the three gene sets (QJ08-2006, QJ10-10, and QJ10-3001) were aligned to genome sequences of the other two isolates using FASTA [29]. As showed in Figure 1, there were as many as 11076 identical genes in these three isolates and contained only a few unique genes among each isolates. There were 277 unique genes in QJ08-2006 and 264 unique genes in QJ10-10, and 213 unique genes in QJ10-3001 (Figure 1). To gain a functional annotation of the unique genes in isolates QJ08-2006, QJ10-10, and QJ10-3001, the gene sets were annotated by predicting secreted proteins. A small number of the secreted proteins were identified from the unique gene set: 16 (5.78\%) in QJ08-2006, 31 (11.74\%) in QJ10-10, 


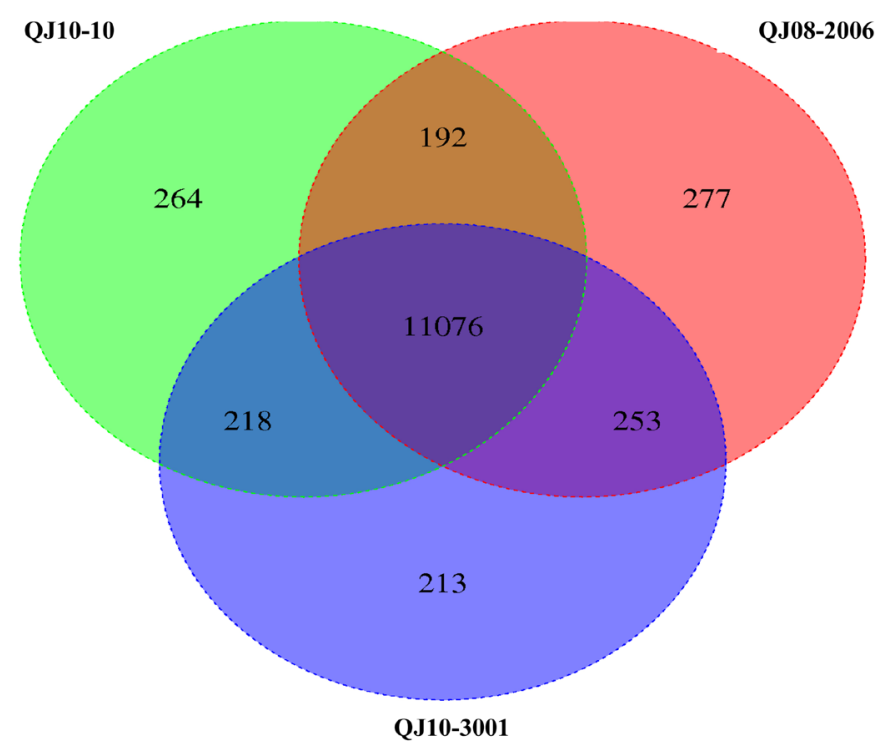

Figure 1. Venn diagram showing the presence/absence variation of the gene set from there sequenced isolates.

and $19(8.92 \%)$ in QJ10-3001, showing that there are few of the secreted proteins in a unique gene set.

\subsection{Analysis of Avirulence Genes in Some Sequenced Magnaporthe}

We had compared $A v r$ genes in some sequenced $M$. oryzae strains including 70-15, QJ08-2006, QJ10-10, and QJ10-3001 (Table 2). The avirulence gene, $A v r-C O 39$ was absent in all of the analyzed strains (Table 2). Avr-Pi9 and $A v r-$ Piz- $t$ were present in all sequenced strains. Avr-Pii was absent in all of the analyzed strains except the strain P131 (Table 2). Compared with QJ10-10, QJ103001, the strain QJ08-2006 has a unique secreted protein gene, AvrPib, while the isolates QJ10-10, and QJ10-3001 had an insertion of a Pot3 element in the promoter of the AvrPib gene, which has led to a gain in virulence towards rice varieties carrying the $R$ gene, $P i b$. We validated in silico analyzed host specificity factors and $A v r$ genes by PCR amplification using gene specific primers [30]. This validation confirmed the accuracy of prediction of the presence and absence of the aforementioned $A v r$ genes in QJ08-2006, QJ10-10, and QJ10-3001 (data not show).

\subsection{Comparative Genome Analysis Based on Coding Genes between Three Re-Sequenced Isolates}

To confirm the relationship between QJ08-2006, QJ10-10, and QJ10-3001, we compared the sequence of the predicted gene and secreted protein genes between three re-sequenced isolates with gene-genome model and Blastn alignment (-max_target_seqs 2-outfit 7, the rest are default), five kinds of variations had been identified, of which were identical (Identity), SNP, small Indel (Sindel), 
Table 2. Presence/absence variation of cloned avirulence ( $A v r$ ) genes among sequenced strains of $M$. oryzae.

\begin{tabular}{|c|c|c|c|c|c|c|c|c|c|c|}
\hline \multirow{2}{*}{$\begin{array}{l}\text { Avirulence } \\
(A v r) \text { genes }\end{array}$} & \multicolumn{2}{|c|}{ M. oryzaestrains } & \multirow[b]{2}{*}{ QJ10-3001 } & \multirow[b]{2}{*}{$70-15$} & \multirow[b]{2}{*}{ Guy11 } & \multirow[b]{2}{*}{ FJ87278 } & \multirow[b]{2}{*}{ Y34 } & \multirow[b]{2}{*}{$\mathrm{P} 131$} & \multirow[b]{2}{*}{$98-06$} & \multirow[b]{2}{*}{ GD-05-029b } \\
\hline & QJ08-2006 & QJ10-10 & & & & & & & & \\
\hline Avr-Pik & $\mathrm{P}$ & $\mathrm{P}$ & $\mathrm{P}$ & $\mathrm{P}$ & $\mathrm{P}$ & $\mathrm{P}$ & $\mathrm{P}$ & A & $\mathrm{P}$ & $\mathrm{P}$ \\
\hline Avr1-CO39 & A & A & $\mathrm{A}$ & $\mathrm{A}$ & A & A & A & A & A & A \\
\hline$A v r-P i 9$ & $\mathrm{P}$ & $\mathrm{P}$ & $\mathrm{P}$ & $\mathrm{P}$ & $\mathrm{P}$ & $\mathrm{P}$ & $\mathrm{P}$ & $\mathrm{P}$ & $\mathrm{P}$ & $\mathrm{P}$ \\
\hline Avr-Pia & A & A & A & A & A & A & $\mathrm{P}$ & $\mathrm{P}$ & A & A \\
\hline$A v r-P i b$ & $\mathrm{P}$ & Partial & Partial & $\mathrm{P}$ & $\mathrm{P}$ & $\mathrm{P}$ & $\mathrm{P}$ & $\mathrm{P}$ & $\mathrm{P}$ & $\mathrm{P}$ \\
\hline Avr-Pii & A & $\mathrm{A}$ & A & A & $\mathrm{A}$ & A & $\mathrm{A}$ & $\mathrm{P}$ & A & A \\
\hline Avr-Pita & $\mathrm{P}$ & A & $\mathrm{P}$ & $\mathrm{P}$ & $\mathrm{P}$ & $\mathrm{P}$ & $\mathrm{P}$ & $\mathrm{P}$ & $\mathrm{P}$ & A \\
\hline Avr-Piz- $t$ & $\mathrm{P}$ & $\mathrm{P}$ & $\mathrm{P}$ & $\mathrm{P}$ & $\mathrm{P}$ & $\mathrm{P}$ & $\mathrm{P}$ & $\mathrm{P}$ & $\mathrm{P}$ & $\mathrm{P}$ \\
\hline
\end{tabular}

Note: $\mathrm{P}$ indicates presence, A indicates absence, Partial indicates partial of avirulence gene can be detected, mostly interrupted by TE insertion in promoter region.

Table 3. Comparative analysis of genes and secreted protein genes between three re-sequenced isolates.

\begin{tabular}{|c|c|c|c|c|c|}
\hline Type of comparative ${ }^{1)}$ & Identity & SNP & Sindel & Lindel & Unmapped \\
\hline \multicolumn{6}{|l|}{ Genes } \\
\hline QJ08-2006 VS QJ10-10 & 7541 & 69 & 4362 & 10 & 11 \\
\hline QJ08-2006 VS QJ10-3001 & 7567 & 63 & 4351 & 11 & 1 \\
\hline QJ10-10 VS QJ08-2006 & 7518 & 72 & 4357 & 7 & 2 \\
\hline QJ10-10 VS QJ10-3001 & 7574 & 48 & 4326 & 7 & 1 \\
\hline QJ10-3001 VS QJ08-2006 & 7535 & 60 & 4347 & 9 & 1 \\
\hline QJ10-3001 VS QJ10-10 & 7557 & 43 & 4334 & 7 & 11 \\
\hline \multicolumn{6}{|l|}{ Secreted protein genes } \\
\hline QJ08-2006 VS QJ10-10 & 1027 & 4 & 371 & 1 & 5 \\
\hline QJ08-2006 VS QJ10-3001 & 1033 & 4 & 369 & 2 & 0 \\
\hline QJ10-10 VS QJ08-2006 & 1013 & 6 & 390 & 1 & 0 \\
\hline QJ10-10 VS QJ10-3001 & 1017 & 6 & 387 & 0 & 0 \\
\hline QJ10-3001 VS QJ08-2006 & 1029 & 5 & 384 & 2 & 0 \\
\hline QJ10-3001 VS QJ10-10 & 1030 & 5 & 378 & 2 & 5 \\
\hline
\end{tabular}

${ }^{1}$ Sindel: Small Indel, the sequence length difference is less than $60 \mathrm{bp}$, Lindel: Large Indel, the sequence length difference is more than $60 \mathrm{bp}$.

Large Indel (Lindel), and not present (Unmapped). As showed in Table 3, about 7500 predicted genes were identified between QJ08-2006, QJ10-10, and QJ10-3001, these three isolates contained 4362, 4351, and 4326 small Indel, respectively. And there were existed several SNP, large Indel, and unmapped sequences between these three isolates. Most of the predicted secreted protein genes were identified, and the re-sequenced isolates contained 371, 369, and 387 small Indel, respectively. The above results indicated that the relationship be- 
tween QJ08-2006, QJ10-10, and QJ10-3001 was very closest.

\section{Discussion}

In several eukaryotic organisms, comparative analysis of multiple genomes of the same species has been used to improve assembly and annotation and to identify genome variations [31] [32] [33]. The rice blast fungus is well-known for its natural genetic variation in the field, resulting in the emergence of new epidemics threatening to world food production [2] [7]. Genomic comparative analyses of multiple genomics in the same pathogens not only could improve the steady of the genome assembly and annotation, and the identification of variations in the plant pathogens' genome, but also open a door to explore new virulence mechanism of the pathogen for effective plant disease control [20]. In this study, we sequenced three field isolates of $M$. oryzae from Southern China. These three field isolates were sampled from the varieties Ma-Ba-Yin-Zhan or Xin-Yin-Zhan, which have been cultivated in the rice area of northern Guangdong for a long time [30]. Genome analysis indicated that these re-sequenced field isolates were much more closely related to each other, the overall genome content and composition were similar among these three isolates, indicated that the rice blast fungus maintained a stable genomic mutation under the pressure of resistant varieties selection.

By the pathotype assays, we found QJ08-2006 was avirulent to the rice variety Xin-Yin-Zhan, while both the strain QJ10-10 and QJ10-3001 were impressively virulent to Xin-Yin-Zhan [30]. Comparative genomics found that the sequenced strains have their unique sequences, suggesting some pathogenic-related sequences may exist outside core genome regions [19]. The whole-genome re-sequenced results showed that these three strains showed quite similar features, e.g., genome size, number of predicted genes, and secreted protein genes, showed that they have a closer relationship with each other. And there were as many as 11,076 identical genes in these three isolates and contained only a few unique genes in each isolate (Figure 1). Among them, a small number of the secreted proteins were identified from the unique gene set: 16 (5.78\%) in QJ08-2006, 31 (11.74\%) in QJ10-10, and 19 (8.92\%) in QJ10-3001, showing few of the secreted proteins present in the unique gene set. These data suggested that little genetic differences exist between the field isolates QJ08-2006, QJ10-10, and QJ10-3001.

Yoshida et al. (2009) observed that the presence or absence of effector gene polymorphisms were often associated with unstable genomic regions near the chromosome ends. It was therefore hypothesized that isolate-specific regions located at the chromosomal ends supplied new effectors and pathogenicity-related factors to drive genome evolution. The dynamic adaptation of $M$. oryzae may be primarily achieved by the deletion or recovery of $A v r$ genes [34]. Such hypotheses indicate that further genome sequencing of multiple $M$. oryzae isolates is warranted for characterizing rice pathogenic strains [16]. Most of the cloned $A v r$ genes of $M$. oryzae reflected the important role of transposable elements (TEs) in 
variations in pathogenicity and the loss of function of $A v r$ genes in $M$. oryzae [14] [21] [35]. In our study, the effector $A v r P i b$ also showed polymorphisms in these three re-sequenced isolates. The isolates QJ10-10, and QJ10-3001 had an insertion of a Pot3 element in the promoter of the AvrPib gene, which has led to a gain in virulence towards rice varieties carrying the $R$ gene, $P i b$ [30]. The Pot3 element inserted in the AvrPib promoter of QJ10-10 and QJ10-3001 suggested this gene is undergoing positive selection, which probably enables these isolates to overcome the resistant rice varieties that harbor $A v r P i b$ alleles recently. The loss of function of $A v r P i b$ was also identified in 300 isolates collected in five different rice-growing areas in China, and a Pot3 insertion in various positions of $-260 \mathrm{bp},-236 \mathrm{bp},-128 \mathrm{bp},-52 \mathrm{bp},-22 \mathrm{bp}$, and $+170 \mathrm{bp}$, leading to the loss of avirulence function of $A v r P i b$ in the five populations [36]. In the 248 virulent isolates collected from a disease hotspot in Philippine, Pot3 was present at the AvrPib region of $-304 \mathrm{bp},-125 \mathrm{bp}$, and $+169 \mathrm{bp}$ in each of avrPib-H1, $-\mathrm{H} 2$, and $-\mathrm{H} 3$ isolates [37]. Similarly, some researchers reported that the diversification in the genome location of Avr-Pita was the consequence of recognition by Pita in rice [12] [34] [38].

To date, only $12 A v r$ genes have been cloned in M. oryzae. By contrast, more than $28 R$ genes in rice controlling race- and not race-specific resistance to $M$. oryzae have been cloned [39] [40]. In the traditional "gene-for-gene" model, resistance $(R)$ genes in the host specifically recognize corresponding avirulence $(A v r)$ genes of the pathogens. Recognition is followed by triggering a hypersensitive response (HR) [41]. Thus, there were a lot of potentials to identify and clone Avr genes in $M$. oryzae. Before 2009, only the $P W L$ family of three members $P W L 1, P W L 3$ and $P W L 4$ were cloned by homology to $P W L 2$ [42], other virulence genes mainly were cloned by map-based cloning, such as $P W L 2, A v r$ Pita, ACE1, Avr1-CO39, Avr-Pizt, Avr-Pia, Avr-Pii, AvrPib [12] [35] [36] [43] [44]. With the rapid development of biotechnology and high-throughput sequencing technology, Avr-Piks/Km/Kp,Avr-Pi9 and Avr-Pi54 were cloned by using the method of comparative genomics. Due to the instability of the rice blast fungus genome, and that $A v r$ proteins lack conserved domains, the mapbased cloning, homology cloning and other traditional cloning strategies were not be effectively utilized. Therefore, whole-genome sequencing of more field blast isolates could accelerate cloning more $A v r$ genes and obtain more information about effector proteins.

Overall, this study provided a systematic genomic analysis of the rice blast field isolates QJ08-2006, QJ10-10, and QJ10-3001, collected from the same field in different years in southern China. Based on the analysis of the three isolates genome, gain, loss and changes of isolate-specific sequences will lead to variances of pathogen virulence. We found that under resistant rice selection pressure for a long time, the genome of avirulent isolate and virulent isolate was very similar, while the changes tend to take place in the genetic variation of avirulence genes corresponding to their resistance genes. 


\section{Acknowledgements}

This work was supported by grants from Natural Science Foundation of Guangdong Province (2020A1515011213) and the National key R \& D project (2016YFD0300707), Modern Agricultural Industry Technology System (CARS-01-32; 2020KJ105), Guangdong Provincial Science and Technology Project (2019B020217003), and Project of Guangzhou Science Plan (202002030001).

\section{Conflicts of Interest}

The authors declare no conflicts of interest regarding the publication of this paper.

\section{References}

[1] Jantasuriyarat, C., Gowda, M., Haller, K., Hatfield, J., Lu, G., Lu, G.D., Stahlberg, E., Zhou, B., Li, H.M., Kim, H., Yu, Y., Dean, R.A., Wing, R.A., Soderlund, C. and Wang, G.L. (2005) Large-Scale Identification of Expressed Sequence Tags Involved in Rice and Rice Blast Fungus Interaction. Plant Physiology, 138, 105-115. https://doi.org/10.1104/pp.104.055624

[2] Valent, B. and Chumley, F.G. (1991) Molecular Genetic Analysis of the Rice Blast Fungus, Magnaporthe grisea. Annual Review of Phytopathology, 29, 443-467. https://doi.org/10.1146/annurev.py.29.090191.002303

[3] Couch, B.C. and Kohn, L.M. (2002) A Multilocus Gene Genealogy Concordant with Host Preference Indicates Segregation of a New Species, Magnaporthe oryzae, from M. grisea. Mycologia, 94, 683-693. https://doi.org/10.1080/15572536.2003.11833196

[4] Zhang, H., Zheng, X. and Zhang, Z. (2016) The Magnaporthe grisea Species Complex and Plant Pathogenesis. Molecuar Plant Pathology, 17, 796-804.

https://doi.org/10.1111/mpp.12342

[5] Ebbole, D.J. (2007) Magnaporthe as a Model for Understanding Host-Pathogen Interactions. Annual Review of Phytopathology, 45, 437-456. https://doi.org/10.1146/annurev.phyto.45.062806.094346

[6] Savary, S., Willocquet, L., Pethybridge, S.J., Esker, P., McRoberts, N. and Nelson, A. (2019) The Global Burden of Pathogens and Pests on Major Food Crops. Nature Ecology \& Evolution, 3, 430-439. https://doi.org/10.1038/s41559-018-0793-y

[7] Talbot, N.J. (2003) On the Trail of a Cereal Killer: Exploring the Biology of Magnaporthe grisea. Annual Review of Microbiology, 57, 177-202. https://doi.org/10.1146/annurev.micro.57.030502.090957

[8] Sesma, A. and Osbourn, A.E. (2004) The Rice Leaf Blast Pathogen Undergoes Developmental Processes Typical of Root-Infecting Fungi. Nature, 431, 582-586. https://doi.org/10.1038/nature02880

[9] Wilson, R.A. and Talbot, N.J. (2009) Fungal Physiology-A Future Perspective. Microbiology, 155, 3810-3815. https://doi.org/10.1099/mic.0.035436-0

[10] Dean, R.A., Talbot, N.J., Ebbole, D.J., Farman, M.L., Mitchell, T.K., Orbach, M.J., Thon, M., Kulkarni, R., Xu, J.R., Pan, H., Read, N.D., Lee, Y.H., Carbone, I., Brown, D., Oh, Y.Y., Donofrio, N., Jeong, J.S., Soanes, D.M., Djonovic, S., Kolomiets, E., et al. (2005) The Genome Sequence of the Rice Blast Fungus Magnaporthe grisea. Nature, 434, 980-986. https://doi.org/10.1038/nature03449

[11] Kim, S., Park, J., Park, S.Y., Mitchell, T.K. and Lee, Y.H. (2010) Identification and 
Analysis of in Planta Expressed Genes of Magnaporthe oryzae. BMC Genomics, 11, 104. https://doi.org/10.1186/1471-2164-11-104

[12] Orbach, M.J., Farrall, L., Sweigard, J.A., Chumley, F.G. and Valent, B. (2000) A Telomeric Avirulence Gene Determines Efficacy for the Rice Blast Resistance Gene Pita. Plant Cell, 12, 2019-2032. https://doi.org/10.1105/tpc.12.11.2019

[13] Kumar, J., Nelson, R.J. and Zeigler, R.S. (1999) Population Structure and Dynamics of Magnaporthe grisea in the Indian Himalayas. Genetics, 152, 971-984. https://doi.org/10.1093/genetics/152.3.971

[14] Bao, J., Chen, M., Zhong, Z., Tang, W., Lin, L., Zhang, X., Jiang, H., Zhang, D., Miao, C., Tang, H., Zhang, J., Lu, G., Ming, R., Norvienyeku, J., Wang, B. and Wang, Z. (2017) PacBio Sequencing Reveals Transposable Elements as a Key Contributor to Genomic Plasticity and Virulence Variation in Magnaporthe oryzae. Molecular Plant, 10, 1465-1468. https://doi.org/10.1016/j.molp.2017.08.008

[15] Zhong, Z., Chen, M., Lin, L., Han, Y., Bao, J., Tang, W., Lin, L., Lin, Y., Somai, R., Lu, L., Zhang, W., Chen, J., Hong, Y., Chen, X., Wang, B., Shen, W.C., Lu, G., Norvienyeku, J., Ebbole, D.J. and Wang, Z. (2018) Population Genomic Analysis of the Rice Blast Fungus Reveals Specific Events Associated with Expansion of Three Main Clades. ISME Journal, 12, 1867-1878. https://doi.org/10.1038/s41396-018-0100-6

[16] Yoshida, K., Saitoh, H., Fujisawa, S., Kanzaki, H., Matsumura, H., Yoshida, K., Tosa, Y., Chuma, I., Takano, Y., Win, J., Kamoun, S. and Terauchi, R. (2009) Association Genetics Reveals Three Novel Avirulence Genes from the Rice Blast Fungal Pathogen Magnaporthe oryzae. Plant Cell, 21, 1573-1591. https://doi.org/10.1105/tpc.109.066324

[17] Xue, M., Yang, J., Li, Z., Hu, S., Yao, N., Dean, R.A., Zhao, W., Shen, M., Zhang, H., Li, C., Liu, L., Cao, L., Xu, X., Xing, Y., Hsiang, T., Zhang, Z., Xu, J.R. and Peng, Y.L. (2012) Comparative Analysis of the Genomes of Two Field Isolates of the Rice Blast Fungus Magnaporthe oryzae. PLoS Genetics, 8, e1002869.

https://doi.org/10.1371/journal.pgen.1002869

[18] Ma, L.J., van der Does, H.C., Borkovich, K.A., Coleman, J.J., Daboussi, M.J., Di, Pietro, A., Dufresne, M., Freitag, M., Grabherr, M., Henrissat, B., Houterman, P.M., Kang, S., et al. (2010) Comparative Genomics Reveals Mobile Pathogenicity Chromosomes in Fusarium. Nature, 464, 367-373. https://doi.org/10.1038/nature08850

[19] Chen, C., Lian, B., Hu, J., Zhai, H., Wang, X., Venu, R.C., Liu, E., Wang, Z., Chen, M., Wang, B., Wang, G.L., Wang, Z. and Mitchell, T.K. (2013) Genome Comparison of Two Magnaporthe oryzae Field Isolates Reveals Genome Variations and Potential Virulence Effectors. BMC Genomics, 14, 887. https://doi.org/10.1186/1471-2164-14-887

[20] Dong, Y., Li, Y., Zhao, M., Jing, M., Liu, X., Liu, M., Guo, X., Zhang, X., Chen, Y., Liu, Y., Liu, Y., Ye, W., Zhang, H., Wang, Y., Zheng, X., Wang, P. and Zhang, Z. (2015) Global Genome and Transcriptome Analyses of Magnaporthe oryzae Epidemic Isolate 98-06 Uncover Novel Effectors and Pathogenicity-Related Genes, Revealing Gene Gain and Lose Dynamics in Genome Evolution. PLoS Pathogens, 11, e1004801. https://doi.org/10.1371/journal.ppat.1004801

[21] Wu, J., Kou, Y., Bao, J., Li, Y., Tang, M., Zhu, X., Ponaya, A., Xiao, G., Li, J., Li, C., Song, M.Y., Cumagun, C.J., Deng, Q., Lu, G., Jeon, J.S., Naqvi, N.I. and Zhou, B. (2015) Comparative Genomics Identifies the Magnaporthe oryzae Avirulence Effector AvrP $i 9$ That Triggers $P \dot{9}$-Mediated Blast Resistance in Rice. New Phytologist, 206, 1463-1475. https://doi.org/10.1111/nph.13310

[22] Zhu, K.P., Bao, J.D., Zhang, L.H., Yang, X., Li, Y., Zhu, M.H., Lin, Q.Y., Zhao, A., 
Zhao, Z., Zhou, B. and Lu, G.D. (2017) Comparative Analysis of the Genome of the Field Isolate V86010 of the Rice Blast Fungus Magnaporthe oryzae from Philippines. Journal of Integrative Agriculture, 16, 60345-30357. https://doi.org/10.1016/S2095-3119(16)61607-6

[23] Fan, G., Zhang, K., Huang, H., Zhang, H., Zhao, A., Chen, L., Chen, R., Li, G., Wang, Z. and Lu, G.D. (2017) Multiprotein-Bridging Factor 1 Regulates Vegetative Growth, Osmotic Stress, and Virulence in Magnaporthe oryzae. Current Genetics, 63, 293 309. https://doi.org/10.1007/s00294-016-0636-9

[24] Al-Samarrai, T.H. and Schmid, J. (2000) A Simple Method for Extraction of Fungal Genomic DNA. Letters in Applied Microbiology, 30, 53-56. https://doi.org/10.1046/j.1472-765x.2000.00664.x

[25] Kurtz, S., Phillippy, A., Delcher, A.L., Smoot, M., Shumway, M., Antonescu, C. and Salzberg, S.L. (2004) Versatile and Open Software for Comparing Large Genomes. Genome Biology, 5, R12. https://doi.org/10.1186/gb-2004-5-2-r12

[26] Sonnhammer, E.L., von Heijne, G. and Krogh, A. (1998) A Hidden Markov Model for Predicting Transmembrane Helices in Protein Sequences. Proceedings International Conference Intelligent Systems Molecular Biology, Vol. 6, 175-182.

[27] Petersen, T.N., Brunak, S., von Heijne, G. and Nielsen, H. (2011) SignalP 4.0: Discriminating Signal Peptides from Transmembrane Regions. Nature Methods, 8, 785786. https://doi.org/10.1038/nmeth.1701

[28] Slater, G.S. and Birney, E. (2005) Automated Generation of Heuristics for Biological Sequence Comparison. BMC Bioinformatics, 6, 31.

https://doi.org/10.1186/1471-2105-6-31

[29] Pearson, W.R. (1996) Effective Protein Sequence Comparison. Methods in Enzymology, 266, 227-258. https://doi.org/10.1016/S0076-6879(96)66017-0

[30] Wang, W., Su, J., Chen, K., Yang, J., Chen, S., Wang, C., Feng, A., Wang, Z., Wei, X., Zhu, X., Lu, G.D. and Zhou, B. (2021) Dynamics of the Rice Blast Fungal Population in the Field after Deployment of an Improved Rice Variety Containing Known Resistance Genes. Plant Disease, 105, 919-928. https://doi.org/10.1094/PDIS-06-20-1348-RE

[31] Kellis, M., Patterson, N., Birren, B., Berger, B. and Lander, E.S. (2004) Methods in Comparative Genomics: Genome Correspondence, Gene Identification and Regulatory Motif Discovery. Journal of Computational Biology, 11, 319-355. https://doi.org/10.1094/PDIS-06-20-1348-RE

[32] Novo, M., Bigey, F., Beyne, E., Galeote, V., Gavory, F., Mallet, S., Cambon, B., Legras, J.L., Wincker, P., Casaregola, S. and Dequin, S. (2009) Eukaryote-to-Eukaryote Gene Transfer Events Revealed by the Genome Sequence of the Wine Yeast Saccharomyces cerevisiae EC1118. Proceedings National Academy Sciences USA, 106, 16333-16338. https://doi.org/10.1073/pnas.0904673106

[33] Andersen, M.R., Salazar, M.P., Schaap, P.J., van de Vondervoort, P.J., Culley, D., Thykaer, J., Frisvad, J.C., Nielsen, K.F., Albang, R., Albermann, K., Berka, R.M., Braus, G.H., et al. (2011) Comparative Genomics of Citric-Acid-Producing Aspergillus niger ATCC 1015 versus Enzyme-Producing CBS 513.88. Genome Research, 21, 885-897. https://doi.org/10.1101/gr.112169.110

[34] Chuma, I., Isobe, C., Hotta, Y., Ibaragi, K., Futamata, N., Kusaba, M., Yoshida, K., Terauchi, R., Fujita, Y., Nakayashiki, H., Valent, B. and Tosa, Y. (2011) Multiple Translocation of the AVR-Pita Effector Gene among Chromosomes of the Rice Blast Fungus Magnaporthe oryzae and Related Species. PLoS Pathogens, 7, e1002147. https://doi.org/10.1371/journal.ppat.1002147 
[35] Li, W., Wang, B., Wu, J., Lu, G., Hu, Y., Zhang, X., Zhang, Z., Zhao, Q., Feng, Q., Zhang, H., Wang, Z., Wang, G., Han, B., Wang, Z. and Zhou, B. (2009) The Magnaporthe oryzae Avirulence Gene AvrPiz- $t$ Encodes a Predicted Secreted Protein That Triggers the Immunity in Rice Mediated by the Blast Resistance Gene Piz- $t$. Molecular Plant-Microbe Interactions, 22, 411-420. https://doi.org/10.1094/MPMI-22-4-0411

[36] Zhang, S., Wang, L., Wu, W., He, L., Yang, X. and Pan, Q. (2015) Function and Evolution of Magnaporthe oryzae Avirulence Gene AvrPib Responding to the Rice Blast Resistance Gene Pib. Scientific Reports, 5, Article No. 11642. https://doi.org/10.1038/srep11642

[37] Olukayode, T., Quime, B., Shen, Y.C., Yanoria, M.J., Zhang, S., Yang, J., Zhu, X., Shen, W.C., von Tiedemann, A. and Zhou, B. (2019) Dynamic Insertion of Pot3 in AvrPib Prevailing in a Field Rice Blast Population in the Philippines Led to the High Virulence Frequency against the Resistance Gene Pib in Rice. Phytopathology, 109, 870-877. https://doi.org/10.1094/PHYTO-06-18-0198-R

[38] Kanzaki, H., Yoshida, K., Saitoh, H., Fujisaki, K., Hirabuchi, A., Alaux, L., Fournier, E., Tharreau, D. and Terauchi, R. (2012) Arms Race Co-Evolution of Magnaporthe oryzae AVR-Pik and Rice Pik Genes Driven by Their Physical Interactions. Plant Journal, 72, 894-907. https://doi.org/10.1111/j.1365-313X.2012.05110.X

[39] Su, J., Wang, W., Han, J., Chen, S., Wang, C., Zeng, L., Feng, A., Yang, J., Zhou, B. and Zhu, X. (2015) Functional Divergence of Duplicated Genes Results in a Novel Blast Resistance Gene Pi50 at the Pi2/9 Locus. Theoretical and Applied Genetics, 128, 2213-2225. https://doi.org/10.1007/s00122-015-2579-9

[40] Deng, Y., Zhai, K., Xie, Z., Yang, D., Zhu, X., Liu, J., Wang, X., Qin, P., Yang, Y., Zhang, G., Li, Q., Zhang, J., Wu, S., Milazzo, J., Mao, B., Wang, E., Xie, H., Tharreau, D. and He, Z. (2017) Epigenetic Regulation of Antagonistic Receptors Confers Rice Blast Resistance with Yield Balance. Science, 355, 962-965. https://doi.org/10.1126/science.aai8898

[41] Heath, M.C. (1981) A Generalized Concept of Host-Parasite Specificity. Phytopathology, 71, 1121-1123. https://doi.org/10.1094/Phyto-71-1121

[42] Kang, S., Sweigard, J.A. and Valent, B. (1995) The PWL Host Specificity Gene Family in the Blast Fungus Magnaporthe grisea. Molecular Plant-Microbe Interactions, 8, 939-948. https://doi.org/10.1094/MPMI-8-0939

[43] Miki, S., Matsui, K., Kito, H., Otsuka, K., Ashizawa, T., Yasuda, N., Fukiya, S., Sato, J., Hirayae, K., Fujita, Y., Nakajima, T., Tomita, F. and Sone, T. (2009) Molecular Cloning and Characterization of the AVR-Pia Locus from a Japanese Field Isolate of Magnaporthe oryzae. Molecular Plant Pathology, 10, 361-374. https://doi.org/10.1111/j.1364-3703.2009.00534.x

[44] Ribot, C., Césari, S., Abidi, I., Chalvon, V., Bournaud, C., Vallet, J., Lebrun, M.H., Morel, J.B. and Kroj, T. (2013) The Magnaporthe oryzae Effector AVR1-CO39 Is Translocated into Rice Cells Independently of a Fungal-Derived Machinery. Plant Journal, 74, 1-12. https://doi.org/10.1111/tpj.12099 